\title{
The relaxation of two-dimensional rolls in Rayleigh-Bénard convection
}

\author{
M. R. Paul \\ Department of Physics, California Institute of Technology, Pasadena, California 91125 \\ I. Catton \\ Department of Mechanical and Aerospace Engineering, University of California at Los Angeles, \\ Los Angeles, California 90095-1597
}

(Received 19 October 2003; accepted 21 January 2004; published online 2 April 2004)

\begin{abstract}
Large aspect ratio, two-dimensional, periodic convection layers containing a Boussinesq fluid of finite Prandtl number bounded by rigid or free horizontal surfaces are investigated numerically. The fluid equations are solved using both a standard pseudospectral and a Fourier integral method for the time evolution of finite initial perturbations, both random thermal perturbations and localized roll disturbances, into a final equilibrium state. The suggestion that a Fourier integral solution method is required to yield roll relaxation, the two-dimensional process increasing the convection wavelength to values larger than critical, is investigated. Roll relaxation is found for both free-slip and no-slip surfaces using either solution method as long as the initial state is chosen to be of the form of a localized roll disturbance. A wide variety of simulations are performed and roll relaxation is found to be independent of the periodic domain length, weakly dependent on the Rayleigh number and dependent upon the magnitude of the initial localized roll disturbances. (C) 2004 American Institute of Physics. [DOI: 10.1063/1.1669335]
\end{abstract}

\section{INTRODUCTION}

Rayleigh-Bénard convection is a canonical example of a pattern forming system. ${ }^{1}$ A fascinating question is raised by the pattern selection process upon which a convection layer evolves through time to a final steady state. Wave number selection has been well studied with many different mechanisms now well characterized (see, for example, Refs. 2 and $3)$.

In a large convection layer (where the lateral extent is much larger than the depth) it has been found both numerically (in three-dimensional simulations ${ }^{4-6}$ ) and experimentally ${ }^{7-12}$ that in general the wavelength of convection increases as conditions become more supercritical. The process by which this occurs is complicated (possibly involving the effects of multiple incommensurate selection mechanisms), three-dimensional, and involves the complex interaction of many defect structures. This process is not well understood and we do not attempt here to address this difficult question. However, the wavelength of convection rolls also increase in other simpler situations where the wave number selection process appears to be governed by a twodimensional process involving straight parallel convection rolls undergoing a wave number adjustment without breaking the two-dimensionality by a simple roll expansion. We will call roll relaxation the two-dimensional process by which the convection rolls increase in wavelength to values larger than the value at threshold. Investigating this mechanism and understanding for what conditions it exists are the focus of this paper.

Evidence for such a two-dimensional relaxation mechanism can be found in both experiments and numerics. For example, consider the motion of a grain boundary separating two domains of straight parallel rolls that are perpendicular to one another. ${ }^{13}$ The grain boundary acts as a "soft boundary" allowing the convection rolls to continuously vary in wavelength. A similar "soft boundary" is also formed when a domain of straight parallel rolls are bounded by quiescent flow. ${ }^{14}$ In cases such as the two just described the convection rolls are able to undergo a continuous wave number adjustment by simply undergoing a roll expansion or a roll compression. Although each of these flows are clearly threedimensional the actual mechanism of wave number adjustment appears to be at least approximately twodimensional. Experiment has revealed that under these conditions the wavelength of the convection rolls increases, a roll relaxation, with increasing reduced Rayleigh number, $\epsilon$ [where $\epsilon=\left(R-R_{c}\right) / R_{c}$ and $R$ is the Rayleigh number and $R_{c}$ its critical value].

However, many two-dimensional numerical simulations of Rayleigh-Bénard convection have been performed for a variety of boundary conditions that have found that the wavelength of convection decreases as $R$ increases. ${ }^{5,15,16} \mathrm{Re}$ call that full three-dimensional numerical simulations agree with the experimental results. This raises the question, is the roll relaxation a three-dimensional effect or have the twodimensional numerical simulations been inadequate to capture the appropriate physics? This apparent discrepancy led to a numerical investigation of two-dimensional RayleighBénard convection using a Fourier integral solution procedure, for a convection layer initially containing a localized roll disturbance, that was able to generate roll relaxation for the idealistic case of free-slip boundary conditions. ${ }^{17,18} \mathrm{It}$ was suggested that the Fourier integral approach removed the restriction limiting the possible wave numbers, as is the case for periodic geometries, by allowing the convection rolls to continuously expand or compress. An investigation to 
further understand this process of roll relaxation is discussed below.

\section{DISCUSSION}

We have performed full numerical simulations of the two-dimensional fluid and heat equations. The velocity $\mathbf{u}$, temperature $T$, and pressure $p$, evolve according to the Boussinesq equations,

$$
\begin{aligned}
& \sigma^{-1}\left(\partial_{t}+\mathbf{u} \cdot \boldsymbol{\nabla}\right) \mathbf{u}=-\nabla p+R T \hat{z}+\nabla^{2} \mathbf{u}, \\
& \left(\partial_{t}+\mathbf{u} \cdot \boldsymbol{\nabla}\right) T=\nabla^{2} T, \\
& \boldsymbol{\nabla} \cdot \mathbf{u}=0,
\end{aligned}
$$

where $\partial_{t}$ indicates time differentiation, $\sigma$ is the Prandtl number, and $\hat{z}$ is a unit vector in the vertical direction. The equations are nondimensionalized in the standard manner using the layer depth $d$, the vertical diffusion time for heat $\tau_{v}$ $\equiv d^{2} / \kappa$ where $\kappa$ is the thermal diffusivity, and $\Delta T$, as the length, time, and temperature scales, respectively. The lower and upper surfaces are either both no-slip or free-slip and are held at constant temperature and the vertical boundaries are periodic.

The governing equations are solved numerically using both a Fourier-Chebyshev pseudospectral (referred to as the pseudospectral approach) and a Fourier-integral Chebyshev method (referred to as the Fourier Integral approach) which are described in detail elsewhere. ${ }^{19}$

The pseudospectral algorithm employs a standard semiimplicit time discretization with a Fourier decomposition in the horizontal direction (and therefore periodic) and a Chebyshev decomposition in the vertical direction (parallel with gravity). The nonlinear terms are evaluated in physical space in order to avoid computing convolution sums. The numerical method is an extension of the approach developed in Ref. 20 used to study turbulent channel flow.

The Fourier integral method is similar in structure to the pseudospectral method however the horizontal spatial discretization is accomplished through a numerical implementation of Fourier integrals (using a modified Filon's method with cubic interpolation ${ }^{21}$ ). Therefore in this approach the nonlinear terms are calculated in wavespace by evaluating numerically the resulting convolution integrals. However, the Fourier integral solution method is limited by the resolution allowed by the convolution integrals. When spectral peaks become sharper than what is allowed by the finite wave number grid the error in numerically evaluating the convolution integrals become large thus invalidating the solution from this point in time forward. As reported in Ref. 17 this is a major restriction to the simulations and greatly limits the duration of the numerical simulations. We have performed Fourier integral numerical simulations for many different cases including varying the time step and the wave number discretization to ensure consistency of the numerical results and to investigate if it is possible to delay the onset of these large errors. We have concluded that the increase in the amount of computations greatly outweighs the slight increase in simulation duration making this a prohibitive way to extend the calculations. As a result, using the Fourier integral (a)

(b)

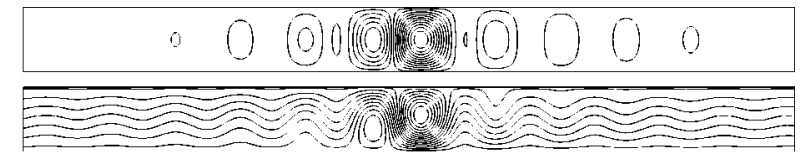

(c)

(d)

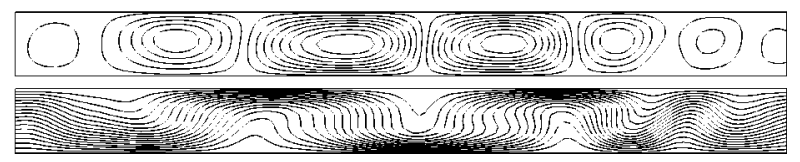

FIG. 1. Simulation flow field starting from a localized roll disturbance using the Fourier integral solution method with free-slip upper and lower boundaries, $\epsilon=3.56, \sigma=0.1$, and $L_{x}=114.9$. Panels (a) and (b) illustrate the initial conditions for $\psi$ and $\theta$, respectively. Panels (c) and (d) illustrate $\psi$ and $\theta$ at time $t=7$ showing that the convection wavelength has increased, the reduced wave number of the central pair of rolls is $q=0.550$ (recall $q=1$ is the pattern wave number at threshold). The field of view is a truncated window of length $L=24$ used for illustrative purposes the entire layer is much larger.

method the final steady solution is not obtainable. The pseudospectral approach, on the other hand, does not suffer from this deficiency and is able to calculate the final steady states.

It should be noted for the Fourier integral approach that although a physical domain length, $L_{x}$, over which the flow field is periodic is not explicitly chosen it is implicity enforced by the inherent wave number discretization, $\Delta k$, chosen when the Fourier integrals are numerically evaluated. Therefore it is not possible to simulate an infinite twodimensional convection layer using a Fourier integral method, actually the calculations are for a periodic layer of length $L_{x}=2 \pi / \Delta k$.

The results of Ref. 17 illustrating two-dimensional roll relaxation are reproduced using both the pseudospectral and the Fourier Integral methods as shown in Figs. 1 and 2. The simulation flow fields are initiated with a localized roll disturbance [see panels (a) and (b) of Fig. 1]. The initial flow field is illustrated by plotting the stream function $\psi$ where $(u, v)=\left(-\partial_{y} \psi, \partial_{x} \psi\right)$ and isotherms of the temperature deviation from the linear conduction profile $\theta$. The localized roll disturbance is composed of a well established pair of convection rolls in the center of the domain that is bounded by nearly quiescent fluid. Following Ref. 17 the localized roll disturbance is constructed from trapezia in Fourier space for $\hat{\psi}$ and $\hat{\theta}$ (where $\hat{\psi}$ and $\hat{\theta}$ are the Fourier transforms of $\psi$ and $\theta$ ). More precisely,

$$
\operatorname{Re}\{\hat{\alpha}\}= \begin{cases}C_{\alpha} \frac{q}{q_{1}} & 0 \leqslant q \leqslant q_{1}, \\ C_{\alpha} & q_{1} \leqslant q \leqslant q_{2}, \\ C_{\alpha} \frac{q_{3}-q}{q_{3}-q_{2}} & q_{2} \leqslant q \leqslant q_{3}, \\ 0 & q_{3} \leqslant q \leqslant q_{\max },\end{cases}
$$

(a)

\section{(b)}

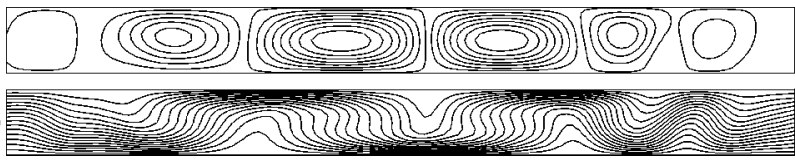

FIG. 2. Simulation flow field at time $t=7$ using the pseudospectral method. Panels (a) and (b) represent $\psi$ and $\theta$, respectively. The reduced wave number of the central pair of rolls is $q=0.550$. The initial conditions and fluid parameters are identical to that of Figs. 1(a) and 1(b). 
TABLE I. Simulation parameters used to generate the initial localized roll disturbance for the free surface simulations represented in Figs. 1 and 2.

\begin{tabular}{cccccc}
\hline \hline$C_{\psi}$ & $C_{\theta}$ & $q_{\max }$ & $q_{1}$ & $q_{2}$ & $q_{3}$ \\
\hline-0.0314 & 0.314 & 3.15 & 0.23 & 2.70 & 2.93 \\
\hline \hline
\end{tabular}

$$
\operatorname{Im}\{\hat{\alpha}\}= \begin{cases}C_{\alpha} & 0 \leqslant q \leqslant q_{2}, \\ C_{\alpha} \frac{q_{3}-q}{q_{3}-q_{2}} & q_{2} \leqslant q \leqslant q_{3}, \\ 0 & q_{3} \leqslant q \leqslant q_{\max },\end{cases}
$$

where $\alpha$ can be either $\psi$ or $\theta$ and the reduced wave numbers (defined as $q=k / k_{c}$, where $k$ is the wave number and $k_{c}$ its value at threshold) $q_{1}, q_{2}, q_{3}, q_{\max }$, and $C_{\alpha}$ are all real constants. The values of $q_{1}$ and $q_{3}$ are chosen to be outside the neutral stability curve. The particular values used in the simulations presented here are shown in Table I.

The aspect ratio of the layer is quite large $L_{x}=114.9$ and only a small window of the layer of length $L=24$ is shown in the figures. The reduced wave number of the central roll pair, defined as $2 \pi / \lambda$, where $\lambda$ is the wavelength of the central roll pair, decreases with time until reaching a value of $q$ $=0.550$ at $t=7$ (for free-slip surfaces $k_{c}=2.22$ ) indicating that the wavelength of the rolls have become larger than the critical value. These results are in agreement with Ref. 17 which reports a value of $q=0.54-0.59$ for these parameters. The numerical solution using the Fourier integral method could not be continued beyond this point because of the errors in calculating the convolution integrals.

The same results are achieved using the pseudospectral approach when starting from identical initial conditions. The resulting stream function, $\psi$, and thermal perturbation fields, $\theta$, are illustrated in Fig. 2 panels (a) and (b) which should be compared with Fig. 1 panels (c) and (d). However, in this case the simulation can be continued until a steady state is reached. When this is done it is found that the convection rolls continue to relax until $q=0.47$ at $t=9.5$ at which point the wave number monotonically increases to a value of $q$ $=0.89$ as the entire layer becomes filled with a periodic field of convection rolls. The smallest value of the wave number of $q=0.47$ at $t=9.5$ is important in that it represents an approximation to the wave number in an infinite domain free of influences from the lateral boundaries. The monotonic decrease in the value of the wave number after $t=9.5$ to its final value of $q=0.89$ is a slow gradual process requiring nearly $t=100$ to occur. The roll relaxation process is independent of the solution method. Since the pseudospectral method is faster and also permits solutions to continue to steady state we find that this method is desirable over the Fourier integral approach.

The aspect ratio of the layer is chosen to be large enough such that the roll relaxation process occurs long before the effects of the lateral sidewalls become important. This is illustrated by plotting the stream function at mid-depth over the entire convection layer as shown in Fig. 3. Panel (a) of Fig. 3 illustrates the stream function for the initial flow field whereas panel (b) shows the stream function at time $t=7$. In (a)

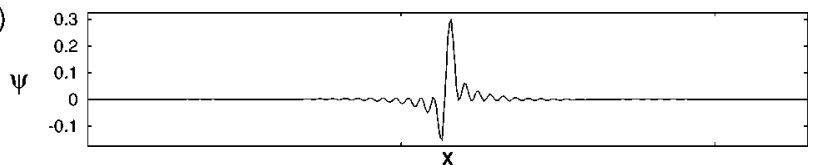

(b)

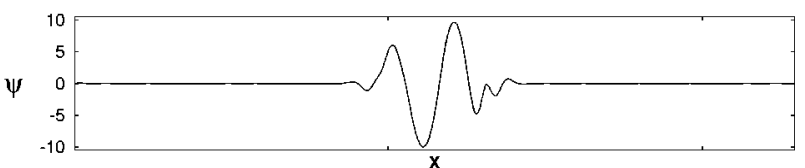

(c)

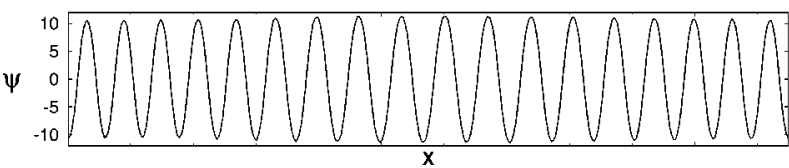

FIG. 3. The stream function $\psi$ over the entire layer $\left(L_{x}=114.9\right)$ for the pseudospectral solution as shown in Fig. 2. Panels (a)-(c) are for $t=0,7$, and 59, respectively. The computational domain is large enough such that the roll relaxation occurs before the lateral boundaries significantly influence the simulation results.

both cases the convection rolls are quite localized in the center of the domain and are not heavily influenced by the lateral boundaries. As illustrated in panel (c) the layer eventually becomes filled with periodic convection rolls. The wave number selection process that occurs after the roll relaxation has occurred is strongly linked with the length of the periodic domain and the particular choice of initial conditions and is not the focus here.

Roll relaxation is also found for the more realistic case of no-slip boundaries (but still periodic in the horizontal direction) for an air filled convection layer using the pseudospectral algorithm as shown in Fig. 4. The roll relaxation occurs on a fairly fast time scale where the central pair of convection rolls is at their smallest value of $q=0.8213$ (where $k_{c}=3.117$ for no-slip boundaries) by the time $t$ $=0.5$. The simulation was allowed to continue until a steady state was reached and at $t=238$ the wave number of the periodic convection layer was $q=1.088$.

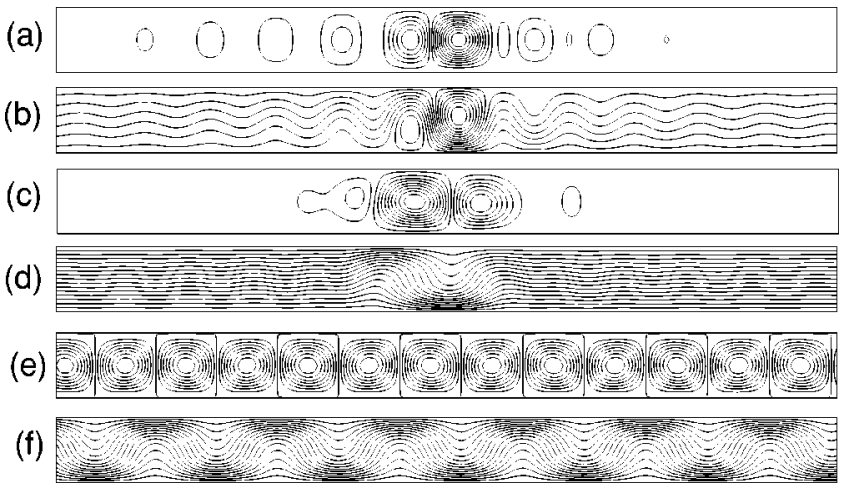

FIG. 4. Simulation flow field starting from a localized roll disturbance for an air filled convection layer with rigid no-slip upper and lower boundaries, $\epsilon=0.522, \sigma=0.7$, and $L_{x}=114.9$. Panels (a) and (b) illustrate the initial conditions for $\psi$ and $\theta$, respectively. Panels (c) and (d) illustrate $\psi$ and $\theta$ at time $t=0.5$ where the wave number of the central pair of rolls is $q$ $=0.821$. Panels (e) and (f) illustrate $\psi$ and $\theta$ at time $t=238$ where the wave number of the central pair of rolls is $q=1.087$. The field of view is a truncated window of length $L=24$ used for illustration. 
TABLE II. Simulation results for three different domain lengths. Simulations are initiated from a localized roll disturbance and are for no-slip boundary conditions, $\epsilon=0.52, \sigma=0.7$. The values of $q$ and $t$ are when the roll is most relaxed, whereas $q_{f}$ and $N$ are the wave number the number of convection rolls at steady state.

\begin{tabular}{ccccr}
\hline \hline$L_{x}$ & $t$ & $q$ & $q_{f}$ & $N$ \\
\hline 114.9 & 0.5 & 0.831 & 1.088 & 62 \\
162.5 & 0.5 & 0.828 & 1.068 & 86 \\
229.8 & 0.5 & 0.828 & 1.052 & 120 \\
\hline \hline
\end{tabular}

To ensure that the time evolution of the central pair is not affected by the choice of the computational domain length, three different domain lengths were investigated and results are presented in Table II. The simulations are for rigid no-slip boundaries as shown in Fig. 4. Note that the domain lengths are not all simple factors of each other. The roll relaxation process has been found to be independent of the length of the domain. This is true as long as the domain is large enough to allow boundary effects to occur after the convection rolls have expanded. On the other hand, the final steady state wave number also depends upon the size of the computational domain. This is because the convection layer must select the final wavelength based upon the number of pairs of rolls that can fit in that particular domain length. The final state is found to be approximately the wave number maximizing the linear growth rate of disturbances. A small but general trend of roll relaxation is also found for simulations of varying reduced Rayleigh number as shown in Table III.

Additionally, many simulations performed from different random initial thermal perturbations did not exhibit roll relaxation and in general yielded steady state wave numbers that maximized the linear growth rate of disturbances. This is expected because the initial perturbations grow exponentially and then can not undergo small wave number adjustment because of the periodic boundary conditions which would require the creation or destruction of a pair of rolls. Preliminary calculations indicate that the extent to which the rolls relax does depend upon the magnitude of the initial local roll disturbances with the general trend being that the more established the initial convection rolls are initially the larger the amount of roll relaxation. Quantifying this has not been done and would require many more simulations. However, the establishment of the roll relaxation mechanism is clear.

TABLE III. Simulation results illustrating wave number variation with increasing Rayleigh number, $R$. Simulations are initiated from a localized roll disturbance and are for no-slip boundary conditions with $\sigma=0.7$. The values of $q$ and $t$ are when the roll is most relaxed, whereas $q_{f}$ and $N$ are the wave number the number of convection rolls at steady state.

\begin{tabular}{lcccc}
\hline \hline \multicolumn{1}{c}{$\epsilon$} & $q$ & $t$ & $q_{f}$ & $N$ \\
\hline 0.0013 & 0.844 & 0.60 & 0.982 & 56 \\
0.054 & 0.844 & 0.58 & 0.982 & 56 \\
0.17 & 0.841 & 0.54 & 1.052 & 60 \\
0.52 & 0.831 & 0.50 & 1.088 & 62 \\
1.34 & 0.805 & 0.38 & 1.123 & 64 \\
\hline \hline
\end{tabular}

\section{CONCLUSION}

The roll relaxation exhibited for the free-slip boundary simulations in Ref. 17 is reproduced here using both a Fourier integral method and a pseudospectral method and is extended to include the more realistic case of rigid no-slip boundaries. The Fourier integral method is not required to realize roll relaxation in two-dimensional simulations. The requirement, in addition to that of sufficient spectral resolution, is that the simulations start from an initial state composed of a localized roll disturbance. All two-dimensional simulations in the past did not find roll relaxation because of the choice of nonlocalized initial conditions such as a field of random thermal perturbations. The relaxation of convection rolls in a two-dimensional periodic layer are found to not depend on the length of the computational domain length, to be weakly dependent upon the Rayleigh number and to also depend upon the magnitude of the initial localized roll disturbances.

\section{ACKNOWLEDGMENTS}

We are grateful to R. E. Kelly, P. H. Roberts, and W. Meechum for helpful discussions.

${ }^{1}$ M. C. Cross and P. C. Hohenberg, "Pattern formation outside of equilibrium," Rev. Mod. Phys. 65, 851 (1993).

${ }^{2}$ I. Catton, "Wave number selection in Bénard convection," Int. J. Heat Mass Transfer 110, 1154 (1988)

${ }^{3}$ M. C. Cross, P. C. Hohenberg, and S. A. Safran, "Wave number selection in Rayleigh-Bénard convection: A numerical study," Physica D 5, 75 (1982).

${ }^{4}$ F. B. Lipps, "Numerical simulation of three-dimensional Bénard convection in air," J. Fluid Mech. 75, 113 (1976).

${ }^{5}$ F. B. Lipps and R. C. Somerville, "Dynamics of variable wavelength in finite-amplitude Bénard convection," Phys. Fluids 14, 759 (1971).

${ }^{6} \mathrm{H}$. Tomita and K. Abe, "Numerical simulation of the Rayleigh-Bénard convection of air in a box of a large aspect ratio," Phys. Fluids 11, 743 (1999).

${ }^{7}$ R. Farhadieh and R. S. Tankin, "Interferometric study of two-dimensional Bénard convection cells," J. Fluid Mech. 66, 739 (1974).

${ }^{8}$ E. L. Koschmieder, Bénard Cells and Taylor Vortices (Cambridge University Press, New York, 1993).

${ }^{9} \mathrm{R}$. Krishnamurti, "On the transition to turbulent convection. Part 1. The transition from two- to three-dimensional flow," J. Fluid Mech. 42, 295 (1970).

${ }^{10}$ B. Martinet, P. Haldewang, G. Labrosse, J. C. Payan, and R. Payan, "Rayleigh-Bénard instability: Experimental study of the wave number selection," in Cellular Structures in Instabilities, edited by J. E. Wesfreid and S. Zaleski (Springer-Verlag, Gif-sur-Yvette, France, 1984), pp. 33-42.

${ }^{11}$ P. L. Silveston, "Wärmedurchgang in waagerechten flüssigkeitsschichten," Forsch. Ing. Wes. 24, 29 (1958); 24, 59 (1958).

${ }^{12}$ G. E. Willis, J. W. Deardorff, and R. C. J. Somerville, "Roll diameter dependence in Rayleigh convection and its effect upon the heat flux," J. Fluid Mech. 54, 351 (1972).

${ }^{13} \mathrm{~A}$. Pocheau and V. Croquette, "Dislocation motion: A wave number selection mechanism in Rayleigh-Bénard convection," J. Phys. (France) 45, 35 (1984).

${ }^{14}$ V. S. Berdnikov, A. V. Getling, and V. A. Markov, "Wave number selection in Rayleigh-Bénard convection: Experimental evidence for the existence of an inherent optimal scale," Exp. Heat Transfer 3, 269 (1990).

${ }^{15}$ J. R. Herring, "Investigation of problems in thermal convection," J. Atmos. Sci. 20, 325 (1963).

${ }^{16}$ Y. Ogura, "A numerical study of wave number selection in finiteamplitude Rayleigh convection,” J. Atmos. Sci. 28, 709 (1971).

${ }^{17}$ A. V. Getling, "Evolution of two-dimensional disturbances in the Rayleigh-Bénard problem and their preferred wave numbers," J. Fluid Mech. 130, 165 (1983). 
${ }^{18}$ A. V. Getling, Rayleigh-Bénard Convection (World Scientific, Singapore, 1998).

${ }^{19}$ M. Paul, "Wave number selection in Rayleigh-Benard convection," Ph.D. thesis, University of California at Los Angeles, 2000.

${ }^{20} \mathrm{P}$. Moin and J. Kim, "On the numerical solution of time-dependent vis- cous incompressible fluid flows involving solid boundaries," J. Comput. Phys. 35, 381 (1979).

${ }^{21}$ W. H. Press, S. A. Teukolsky, W. T. Vetterling, and B. P. Flannery, $\mathrm{Nu}-$ merical Recipes in Fortran: The Art of Scientific Computing (Cambridge University Press, Cambridge, 1992). 